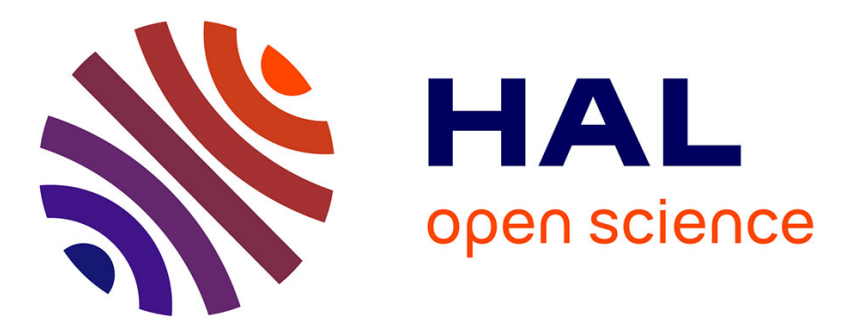

\title{
Fully automatic anatomical, pathological, and functional segmentation from CT scans for hepatic surgery
}

Luc Soler, Hervé Delingette, Grégoire Malandain, Johan Montagnat, Nicholas Ayache, Christophe Koehl, Olivier Dourthe, B. Malassagne, M. Smith, Didier Mutter, et al.

\section{To cite this version:}

Luc Soler, Hervé Delingette, Grégoire Malandain, Johan Montagnat, Nicholas Ayache, et al.. Fully automatic anatomical, pathological, and functional segmentation from CT scans for hepatic surgery. Computer Aided Surgery, 2001, 6 (3), pp.131-42. inria-00615108

\section{HAL Id: inria-00615108 \\ https://hal.inria.fr/inria-00615108}

Submitted on 17 Aug 2011

HAL is a multi-disciplinary open access archive for the deposit and dissemination of scientific research documents, whether they are published or not. The documents may come from teaching and research institutions in France or abroad, or from public or private research centers.
L'archive ouverte pluridisciplinaire HAL, est destinée au dépôt et à la diffusion de documents scientifiques de niveau recherche, publiés ou non, émanant des établissements d'enseignement et de recherche français ou étrangers, des laboratoires publics ou privés. 


\title{
Fully automatic anatomical, pathological, and functional segmentation from CT scans for hepatic surgery
}

\author{
Luc Soler $^{a}(\mathrm{PhD})$, Herve Delingette ${ }^{b}(\mathrm{PhD})$, Gregoire Malandain ${ }^{b}(\mathrm{PhD})$, Johan Montagnat ${ }^{b}(\mathrm{PhD})$, \\ Nicholas Ayache $^{b}(\mathrm{PhD})$, Christophe Koehl $^{a}(\mathrm{E})$, Olivier Dourthe ${ }^{b}(\mathrm{MD})$, Benoit Malassagne ${ }^{a}$ (MD), \\ Michelle Smith $^{a}$ (MD), Didier Mutter ${ }^{a}(\mathrm{MD}, \mathrm{PhD})$, Jacques Marescaux ${ }^{a}$ (MD) \\ Correspondence to Pr. Luc SOLER, \\ aRCAD, 1 place de l'hôpital, 67091, Strasbourg, France \\ Phone: 33388119065 Fax : 33388119099 \\ Email : Luc.Soler@Ircad.u_strasbg.fr \\ Key link : www.virtual-surg.com, www.ircad.org \\ ${ }^{\mathrm{b}}$ Epidaure Project INRIA, BP 93, 06902 Sophia Antipolis France
}

funding:

- EUREKA Master project of the European Community

- La Ligue contre le cancer, comité du Haut-Rhin

- Région Alsace

- Fondation pour la recherche médicale

- IRCAD, Digestive Cancer Research Institute

Article based on Medical Imaging 2000 Image Processing presentation in San Diego

\begin{abstract}
Objective: To improve the planning of hepatic surgery, we have developed a fully automatic anatomical, pathological and functional segmentation of the liver derived from a spiral CT scan.

Materials and methods: From a $2 \mathrm{~mm}$ thick enhanced spiral CT scan, a first stage automatically delineates skin, bones, lungs, kidneys and spleen, by combining the use of thresholding, mathematical morphology and distance maps. Next, a reference 3D model is immerged in the image and automatically deformed to liver contours. Then an automatic gaussians fitting on the imaging histogram estimates the intensities of parenchyma, vessels and lesions. This first result is next improved through an original topological and geometrical analysis, providing an automatic delineation of lesions and veins. Finally, a topological and geometrical analysis based on medical knowledge provides hepatic functional information invisible in medical imaging: portal vein labeling and hepatic anatomical segmentation according to the Couinaud classification.

Results: Clinical validation performed on more than 30 patients shows that this method's delineation of anatomical structures is often more sensitive and more specific than manual delineation by a radiologist.

Conclusion: This study describes the methodology used to create the automatic segmentation of the liver with delineation of important anatomical, pathological and functional structures from a routine CT scan. Using the methods proposed in this study, we have confirmed the accuracy and utility of the creation of 3 - dimensional liver model when compared with the conventional reading of the CT scan by a radiologist. This work, may allow an improvement in preoperative planning of hepatic surgery by more precisely delineating liver pathology and its relation to normal hepatic structures. In the future this data may be integrated with computer-assisted surgery and thus represents a first step towards the development of an augmented reality surgical system.
\end{abstract}

Keywords: segmentation, gaussians fitting, mathematical morphology, discrete topology, labeling, hepatic surgery

Keylink: www.virtual-surg.com, www.ircad.org 


\section{INTRODUCTION}

One of the major goals of computerized medical imaging analysis is to automatically detect, identify and delineate anatomical and pathological structures in 3D medical images. 3D modeling of these structures then allows for easier and more extensive visualization and exploitation of images. In hepatic surgery, medical imaging is used to detect and localize hepatic lesions and their relationship to vascular structures, especially the portal vein that defines the hepatic functional anatomy consisting of several anatomical segments $^{1,2}$. There are several different definitions for dividing the liver into functionally meaningful parts that represent the resection unit. Different authors have proposed the division of the liver into two hemilivers, or into four segments based on the Goldsmith and Woodburne definition ${ }^{3}$ or into eight subsegments based on the Couinaud definition ${ }^{4}$ which is today considered the international standard ${ }^{1}$.

In order to detect lesions and to localize vascular networks defining the anatomical segments, radiologists currently use helical Computed Tomography scan images with intravenous contrast infusion (helical CTI). In these images, tumors appear as dark nodules within bright hepatic tissues whereas vessel trees appear as a network brighter than the liver parenchyma. However, detection of the lesion or localization of the vessels is often difficult to process due to a variable image contrast between liver parenchyma and vessels, and also due to an important image anisotropy, the slice thickness being three times larger than the pixel width.

Therefore in hepatic surgery, one of the goals of computerized medical imaging processing is to automatically delineate liver, lesions, vessels and anatomical segments from the imaging studies. Several authors proposed to delineate the liver contours from CTI images with an automatic ${ }^{5,6,7,8,9}$, or semiautomatic process ${ }^{10}$. Several methods use a deformable model, either to directly delineate structures ${ }^{5,7}$, or to improve the results of a previous delineation technique ${ }^{6}$. In addition, vascular tree segmentation has been performed in different studies ${ }^{11,12,13,10}$. Among these works, the method of Zahlten et al. ${ }^{12,13}$ allows extraction of the portal vein from abdominal CT-scan images, using a region growing technique. This technique has the advantage of giving a topological information about the venous tree, which is useful for building all anatomical segments ${ }^{14}$. However, since it requires a manually-set threshold and an initial seed point, this technique is not fully automatic. Finally, there have been very few studies ${ }^{15,16}$ about the hepatic lesion delineation, sometimes performed by the same methods used to isolate other anatomical structures $^{7}$.

Among all these studies, the work of Gao et al. ${ }^{6}$ is best suited for hepatic surgery planning since it provides a general solution allowing the delineation of the hepatic anatomy, even if the vascular system may not be clearly delineated. But this method of liver segmentation does not provide good results with a liver which contains a large sub-capsular tumor. Also, the work of the MEVIS team ${ }^{12,13,14}$ performs portal vein labeling and anatomical segments delineation, but it always reconstructs eight sub-segments even if the patient has a different number of segments. Moreover, this segmentantion technique requires many time-consuming interactions.

In this article, we propose an original three step anatomical segmentation method, based on the translation of anatomical knowledge into topological, geometrical and morphological constraints. This method allows thus for automatic extraction of liver, hepatic vessels, hepatic lesions and also of the anatomical segments with respect to the three most common definitions: hemilivers, Goldsmith and Woodburne definition and Couinaud definition.

\section{AUTOMATIC LIVER, LESIONS AND VEINOUS SYSTEMS DELINEATION}

\subsection{Patients dataset}

This study has been performed on a set of 35 CT-scans with slices from $2 \mathrm{~mm}$ to $3 \mathrm{~mm}$ of thickness, acquired after contrast agent injection at portal phase, from an helical Siemens Somatom 4 plus CT-scan. The database is composed of 33 images with intravenous injection, and two portoscans. It includes 
healthy subjects, patients with lesions (cyst or tumors), and patients after segmentectomy. Furthermore, the rate of contrast product infiltration into hepatic venous systems is quite variable from one patient to another, due to a difficult evaluation of the portal time.

\subsection{First stage: skin, lungs, bones, kidneys, spleen and liver delineation and image improvement}

This first stage of our method automatically extracts step by step, the skin, lungs, bones, kidneys, the spleen and the liver of a patient, from a CT-scan image. Our method consists in translating anatomical information obtained by the medical imaging and transforming this information by the way of several simple intensities, morphological, topological and geometrical constraints. The intensity in Hounsfield units of air, fat tissue, water and bones are known and are respectively $-1000 \mathrm{HU},-120 \mathrm{HU}$ to $-80 \mathrm{HU}, 0$ $\mathrm{HU}$, and $500 \mathrm{HU}$ to $3000 \mathrm{HU}$. Air is mainly outside the patient and in the lungs (some air may be eventually found into the digestive system too). Isolating the air allows us to easily extract the skin and the lungs boundaries. A simple threshold does not allow for isolating the bones. Because of the contrast agent, others structures, such as the aorta, appear bright. To overcome this, we first isolate the fat tissue (thresholding followed by morphological operation). The bones are then characterized as the brightest structures close to the fat tissue.

Kidney and spleen delineation is more difficult due to their intensity variation. We then propose a solution based on the gray-level histogram analysis of the image limited to regions including the spleen and kidneys. Indeed, the right inferior quarter of the image contains essentially a part of the liver and the right kidney, whereas the left inferior quarter of the image contains only the right kidneys and the spleen. Thus, a comparative analysis of the gray-level histograms allows us to find the intensity range of kidneys, spleen and liver parenchyma, identically localized on both histograms. We then delineate the kidneys and the spleen by performing a thresholding followed by morphological operators.

After all of these anatomical structures are removed from the original image, we finally extract liver. From several existing methods, we chose to use the Montagnat and Delingette method $^{5}$ who proposed an hybrid deformation framework that consider the global transformations computed in the registration framework $^{17}$ as a deformation field similar to the local deformation field of the deformable models ${ }^{18,19}$ scheme. This method applies thus to each $S(i)$ vertex of the model with a locality parameter $l$, a combined force $f(i)$ :

$$
f(i)=(1-l) * \text { GlobalForce }(i)+l * \text { LocalForce }(i)
$$

It is possible to apply to the model in this single framework completely local $(=1)$, completely global $(l=0)$ as well as any intermediate $(0<l<1)$ force between those two ends. This framework introduces a global constraint in the deformation process that may be scale through the $l$ parameter. It makes models more reliable: they are less noise and outliers sensitive. Moreover, the geometric quality of meshes produced by the deformation scheme is better.

Application of this method to liver delineation first requires the initialization of a $3 \mathrm{D}$ reference model in the image. In order to obtain a liver contour by deformation, it is easier and more reliable to use an initial model with a similar shape. This model is a liver template computed onto visible human data of the National Library of Medicine. Delineation is then composed of several stages. Fig. 1 and 2 respectively represent the liver template being bent along each stage and its cut superimposition onto on of the CT scan slides.

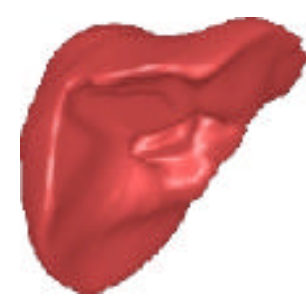

(a) initialization

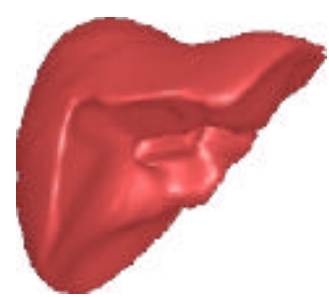

(b) rigid and affine registration

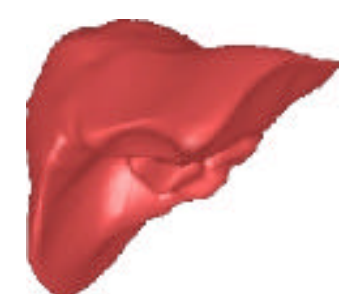

(c) hybrid deformation $l$ low

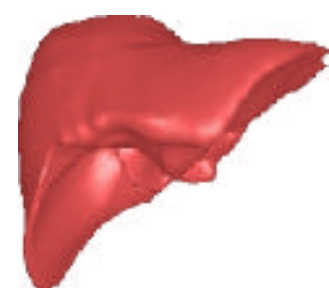

(d) hybrid deformation $l$ high

Figure 1. Shape evolution of the model along stages. 


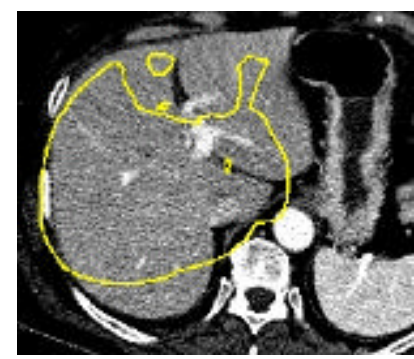

(a) initialization

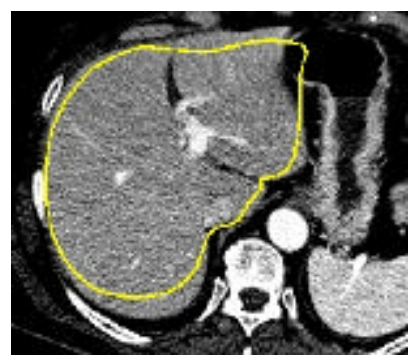

(b) rigid and affine registration

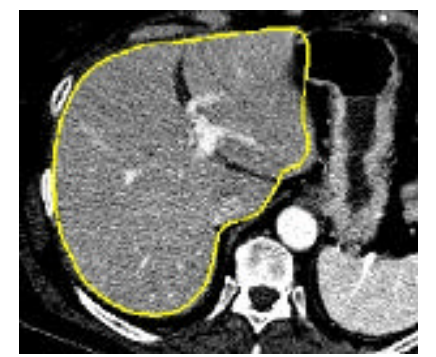

(c) hybrid deformation $l$ low

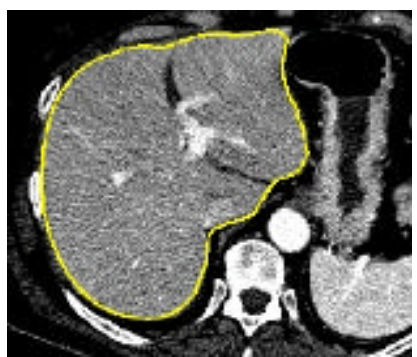

(d) hybrid deformation $l$ high

Figure 2. Evolution of the model cut on one slice of the 3D image.

From the resulting liver delineation, we chose to reduce and improve the initial image in order to speed the process and also to improve the lesions and vessels delineation. Firstly, the extracted liver is used as a mask, which reduces the initial image to the region of interest of the liver. Secondly, the reduced image is filtered with the anisotropic diffusion detailed in ${ }^{20}$. It then reduces the textured aspect of CT scan without loss of structure borders. As shown on Fig. 3, the textured aspect of the initial image is changed in homogenous intensity areas, whereas borders separating parenchyma, vessels and dark areas are preserved.
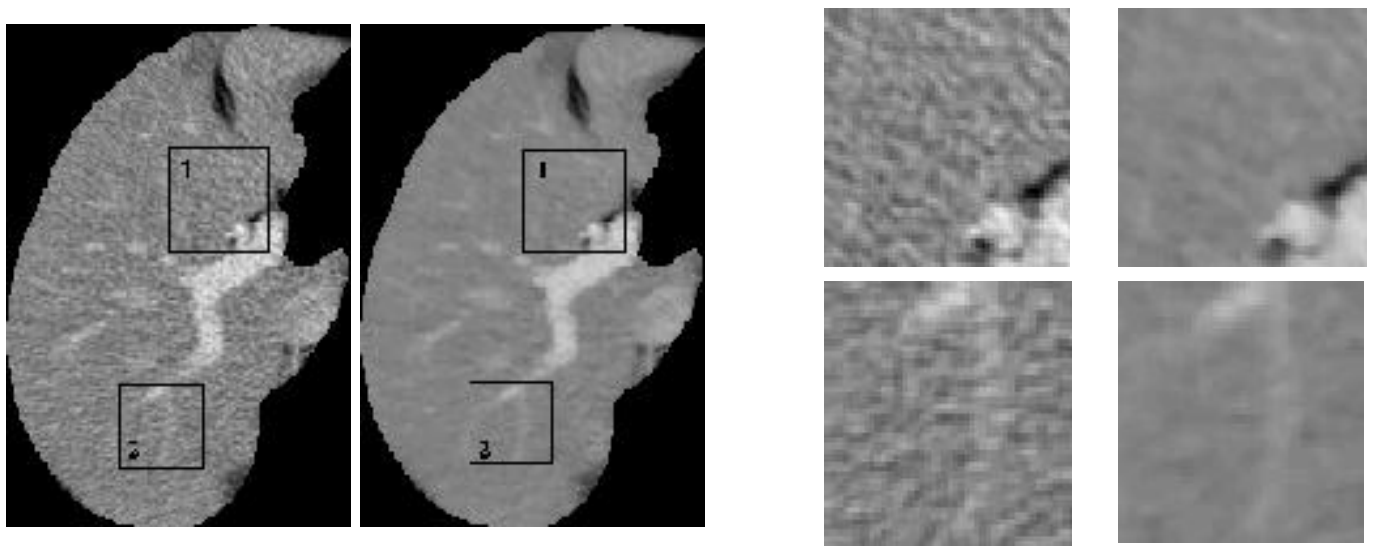

Figure 3. Reduced image before and after anisotropic diffusion, with zoom on two areas: area 1 (up) and area 2 (down)

\subsection{Second stage : automatic delineation of lesions and vascular systems}

We saw previously that Gao et al. ${ }^{6}$ proposed a classification method of all internal structures of the liver. To do this, the authors estimated the intensity distributions of three tissue classes, lesions, parenchyma, and vessels, as trapezoidal functions and used the percentage of voxels belonging to each class for the visualization. We chose to modify their method by considering that the distributions of the same three tissue classes follow a normal law, these distributions being then used to consider thresholds allowing segmentation for each structure. The fitting of distribution onto the gray level histogram is performed by the Levenberg and Marquardt's method ${ }^{21}$, which minimizes a least square criterion and which is currently used for other organs in many articles ${ }^{22,23,24,25}$.

In the liver case, the major limitation of this method is the need to obtain a good initialization of the distribution parameters whereas only the peak corresponding to the liver is usually visible. To fill this handicap, we propose an original resolution in two stages. The first stage fits, on the gray-level histogram, the gaussian curve that corresponds to the parenchyma whose peak is always visible. The subtraction of the resulting gaussian from the histogram provides thus distribution of points that do not belong to the liver (with the some errors close to the first adjustment). From this subtraction, the second stage initializes the two last gaussians and fits the three class gaussians on the initial gray-level histogram. The thresholds are then estimated as the intensities for which two neighbor gaussians cross, defining thus $S_{L F}$, the threshold separating the lesions voxels from the liver voxels, and $S_{F V}$, the threshold separating the liver voxels from the vessels voxels (Fig. 4). 


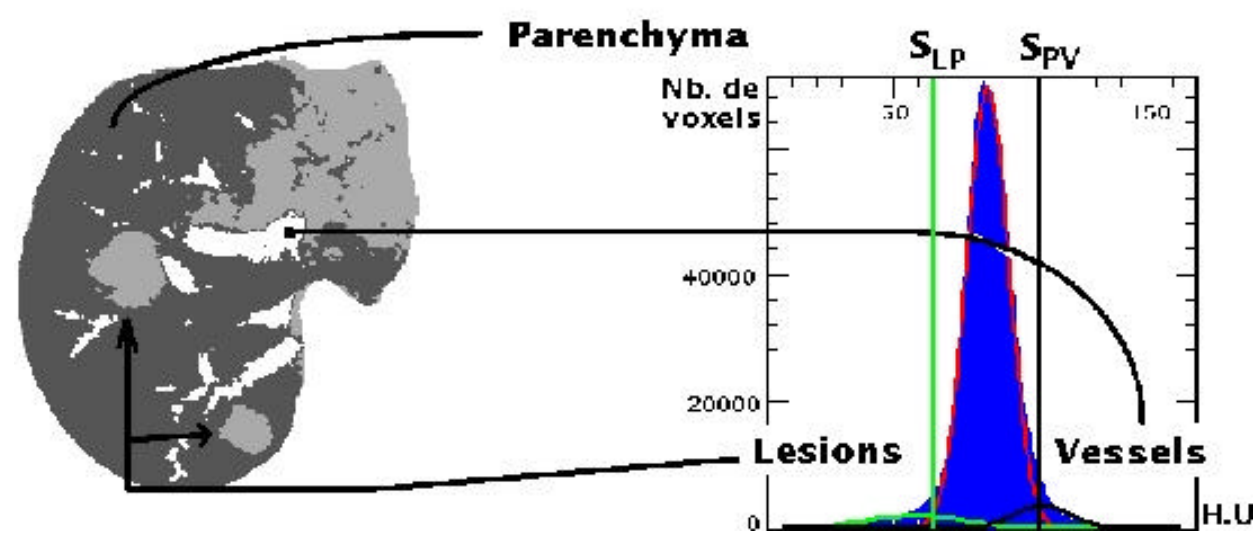

Figure 4. Classification result (left) from thresholding following the three gaussians fitting on the image histogram (right)

This simple thresholding implies misclassification of the voxels of one class being over the gaussian crossing of the neighboring class. So, the intensity information is not enough to obtain a satisfactory delineation of anatomical and pathological structures. In order to improve this result, we have developed original methods based on the translation of anatomical information into topological and geometrical constraints which removes the misclassification of thresholding.

\subsubsection{Lesions Delineation}

Our goal is to remove false positive of thresholding in order to extract all lesions of only 3-mm of thickness. In practice, radiologists limit their analysis to lesions over 5-mm of thickness, but we chose to reduce this minimal thickness in order to improve the reliability of the method. From Gao et al. ${ }^{6}$ work that characterized the lesions by a nodular shape modeled in $2 \mathrm{D}$ by an ellipse, we chose to characterize this form in 3D by an ellipsoid (Fig. 5).
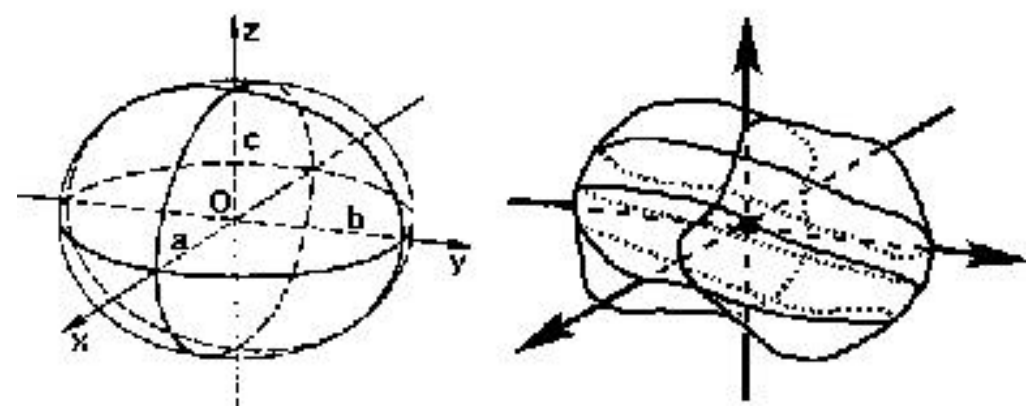

Figure 5. Modeling of a lesion by an ellipsoid: Definition of ellipsoid axes (left) from inertia axe of the real lesion (right)

From this characterization, the radius $\left(r_{1}, r_{2}, r_{3}\right)$ of the ellipsoid associated with each structure can be easily evaluated through the computation of each inertia moments $\left.l_{1}, l_{2}, l_{3}\right)$ corresponding to the axes of inertia $\left(\lambda_{1}, \lambda_{2}, \lambda_{3}\right)$, with the following formula:

$$
r_{i}=\sqrt{\frac{5 \lambda_{i}}{\mu_{000}}} \quad \mu_{000}=\text { Number of voxels of the component }
$$

From these radius computed onto each connected components, we characterize a nodular shape by two ratios $R_{1}$ and $R_{2}$ (Eq. 3), respectively representing the lengthening of the structure and compactness of the ellipsoid associated to the initial structure. We fixed maximal lengthening $R_{1}$ at 2.5 , ensuring thus that the structure will not be overly lengthened and the rate of minimal filling $R_{2}$ to $80 \%$, ensuring thus that the structure will be quite compact.

$$
R_{\mathrm{l}}=\frac{\text { biggestray }}{\text { smallest ray }} \quad R_{2}=\frac{\text { inscribed ellipsoid volume }}{\text { ellipsoid volume }}
$$


This shape is however not found for all lesions. Indeed, some lesions localized at the periphery of the liver in a 5-mm of thickness sub-capsular band, have a flattened shape surfaces with a nodular shaped nucleus ${ }^{26}$. On this kind of structure, morphological erosion can easily extract the central nucleus, which then allows for the computation of the same $R_{1}$ and $R_{2}$ ratios. We chose to keep the maximal lengthening $R_{l}$ at 2.5 but to increase the rate of minimal filling $R_{2}$ to $90 \%$ in order to be more reliable on the peripheral structures of which the number of false positive is more frequent.

All of this information allows for removing false positives from the connected components of the lesion class through three simple stages. The first stage realizes a morphological opening with a $1.5-\mathrm{mm}$ of radius, which removes all components with a thickness under $3-\mathrm{mm}$. The second stage computes the $R_{I}$ and $R_{2}$ ratios on all resulting connected components, and removes those that are not within maximal lengthening and minimal filling constraints. The third stage realizes a morphological erosion on all rejected components localized liver in the 5-mm of thickness sub-capsular band. It then computes the $R_{l}$ and $R_{2}$ ratios on all resulting connected components, and removes those that are not within maximal lengthening and minimal filling constraints. Finally, constraints allow for extracting lesion shaped structures from connected components extracted by the initial thresholding.

\subsubsection{Portal vein delineation}

In regard to the vessels, the thresholding provides two kinds of misclassification: the vessel voxels classified in the liver class, the false negative, and the liver voxels classified in the vessel class, the false positive. It is also important to notice that the different hepatic vascular networks are all grouped together while distinction of the portal vein, hepatic vein and hepatic artery is useful information for surgical planning. We propose here a new method firstly to correct the false negative and secondly to remove the false positive and to distinguish the different vascular networks.

The false negative correction functions by adding vessel voxels classified in the liver class into the vessel class. To accomplish this, two simple solutions can be performed. A first solution, the decreasing of $S_{F V}$ threshold, removes the false negative, but at the same time it increases the number of false positives. A second solution performs a morphological closing on the thresholded image of vessels, but it also adds new false positives (Fig. 6).

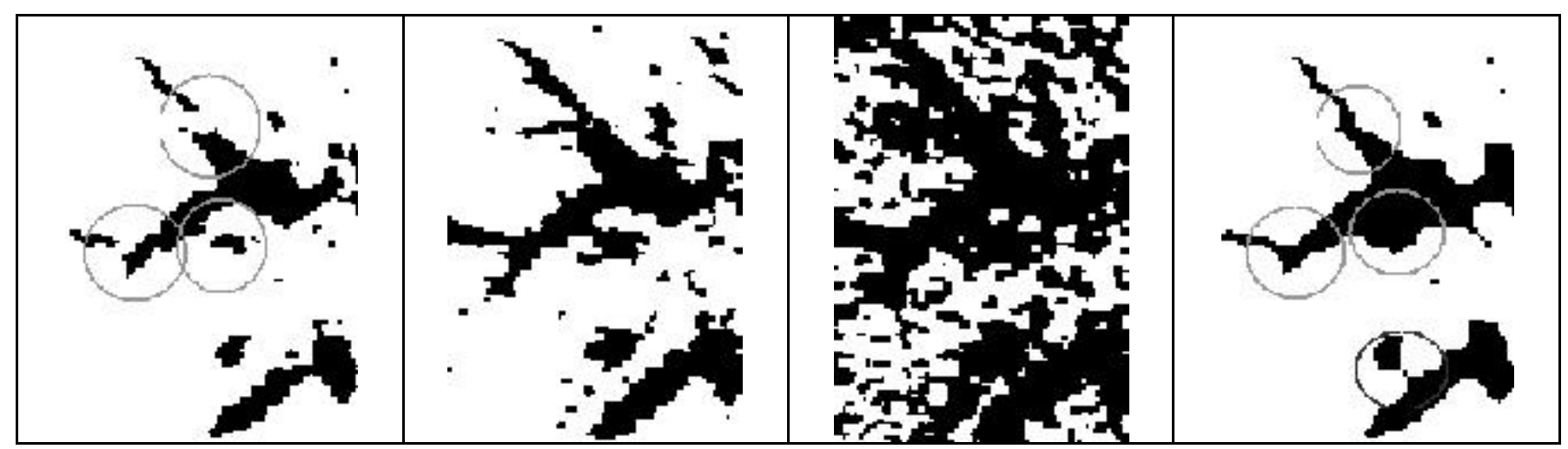

Figure 6. Zoom on one slice of the image : after a thresholding with $\mathrm{S}_{\mathrm{FV}}$ value (left), after a thresholding with the $S_{\mathrm{FV}}$ threshold decreased respectively with $10 \mathrm{HU}$ and $20 \mathrm{HU}$ (middle), and after a morphological closing onto the $S_{\mathrm{FV}}$ thresholded image(right).

We propose to combine these two approaches by adding, in the thresholded image, the voxels resulting from a morphological closing and whose intensity is higher than a given threshold. This threshold is calculated according to the distance $D$ with the voxels resulting from the initial thresholding. Indeed, the closer the point is to these voxels, the more likely it represents the vessels. Therefore, the threshold can be chosen far away from the initial threshold. Conversely, the farther away a point is from these voxels, the more likely it derives from the parenchyma. Therefore, the threshold must be selected close to the initial threshold. We translate this property by the Eq. (4) that allows for computing four $S_{D}$ thresholds from the estimated density distributions of the parenchyma $f_{P}$ and of the vessels $f_{V}$ (Fig. 7). This original hybrid 
method makes it possible to limit the adverse effects of each approach taken independently while correcting false negatives, allowing thus to reconnect branches disconnected by the initial thresholding (Fig. 8).

$$
\frac{f_{P}\left(S_{d}\right)}{f_{V}\left(S_{d}\right)}=\frac{12}{d}
$$
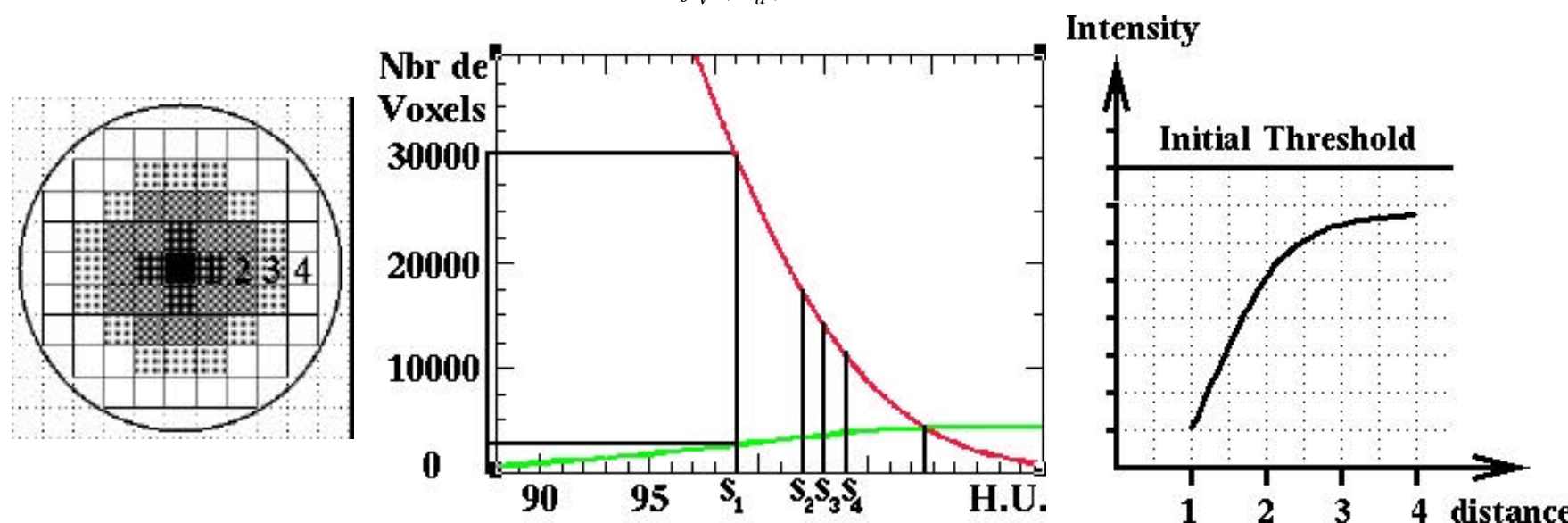

Figure 7. A distance map from voxels resulting to the initial thresholding (left) and the estimated distribution of parenchyma intensities and vessels intensities (middle), combined to the Eq. (4) allows to define four thresholds (right).
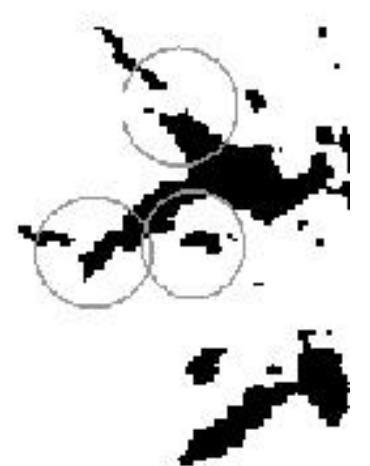
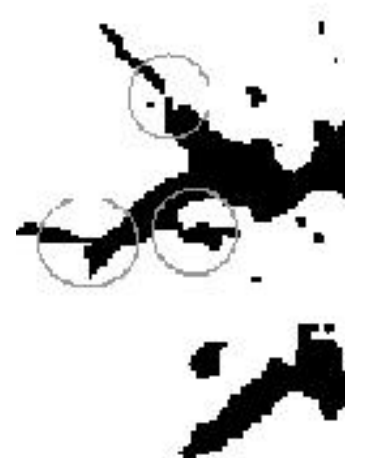

Figure 8. Result of the hybrid method combining morphological closing, distance analysis and thresholding.

The false negative correction being performed, the second stage consists of removing false positives and to distinguish the different vascular networks, which corresponds to four cases illustrated on Fig. 9.

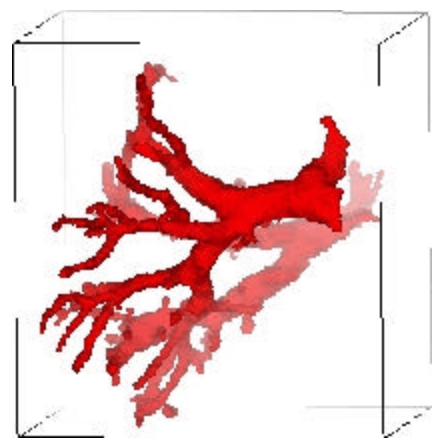

(a)

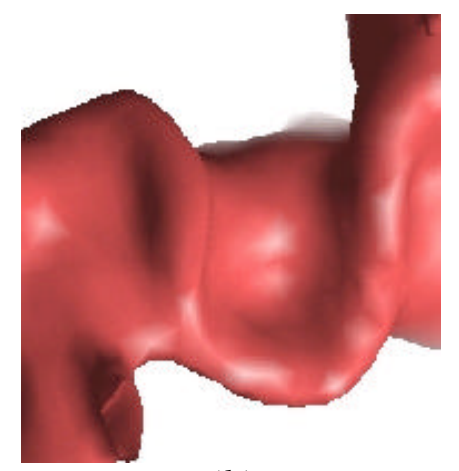

(b)

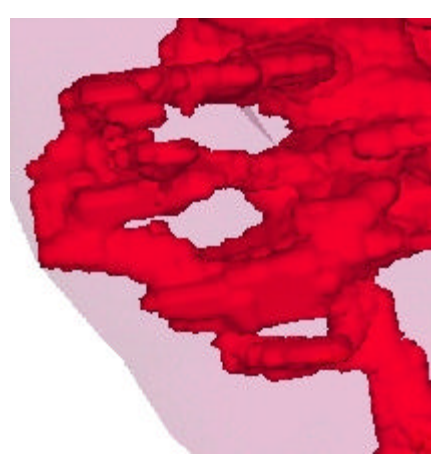

(c)

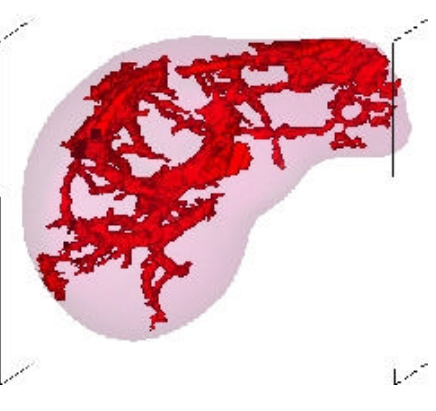

(d)

Figure 9. Four mistake cases: (a) misconnection between portal vein and hepatic vein, (b) misconnection between portal vein and hepatic artery, (c) misconnection creating a loop in the portal vascular network, (d) misclassification of liver voxels in the vessels class (false positive).

The portal vein is tree shaped with several simple properties: wo loops, the reduction in-depth thickness of the branches in the blood flow direction and the absence of obtuse angles in branch junctions. We propose to use these geometrical and topological properties as constraints in a vascular network covering. We first simplify the vascular network by computing its skeleton, as in Zahlten et al. ${ }^{12,13}$, but with the 
Malandain and Bertrand method ${ }^{27,28}$ that provides a skeleton geometrically and topologically much more precise than the region-growing method. Although geometrically and topologically correct, the resulting skeleton has three principle drawbacks: the line irregularity due to image sampling, the barbule presence (small branches) due to skeletisation of the irregular contour shape, and the multiplicity of neighboring junction points. The correction of these drawbacks consists in reducing the skeleton by smoothing lines, rejecting barbules and fusing neighboring junction points. This first treatment allows then for disconnecting the hepatic arterial network from the portal network ( Fig. 10).
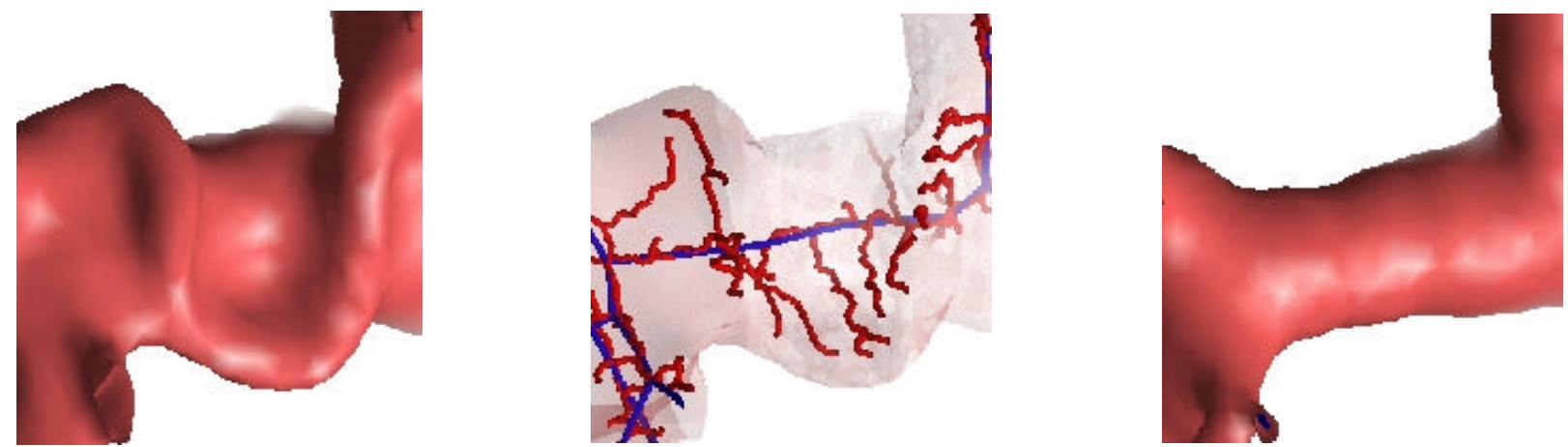

Figure 10. The initial hepatic arterial network (U shaped on left) creates a set of barbules (small skeleton branches in middle). The erasing of barbules allows then for disconnecting arterial branches.

From this skeleton, we cover the network in width from the portal trunk to the smallest branches. The portal trunk is located automatically by its anatomical position characterized by antero-posterior ratio, one of the most stable biometric variables in the human body ${ }^{4}$. At each junction, we can easily analyze the median branch thickness, the angle of branches bifurcation or crossing and loop forming. The system can then automatically remove branches that create loops, branches with a significant thickness increase (higher than $2 \mathrm{~mm}$ ) and branches with a too obtuse angle. The upper limit value of the obtuse angle obtained has been determined in order to removed connections between portal branches and hepatic vein branches. These artificial connections are due to the well known partial volume effect. Two principle cases have been thus defined : Tangency and crossing (Fig. 11). The tangency is defined by a minimal angle of $135^{\circ}$ between two branches $B 1$ and $B 2$ with a same origin $J$. The branch $B J$ is then removed. The crossing case follows the same idea. It is defined when almost 2 of 3 or more branches of a crossing have a angle over $135^{\circ}$. In the example case of figure $11, B 2$ and $B 3$ have a angle $A 2-3$ over $135^{\circ} . B J$ and $B 1$ having an angle $A J-1$ over $135^{\circ}, B 2$ and $B 3$ are removed.

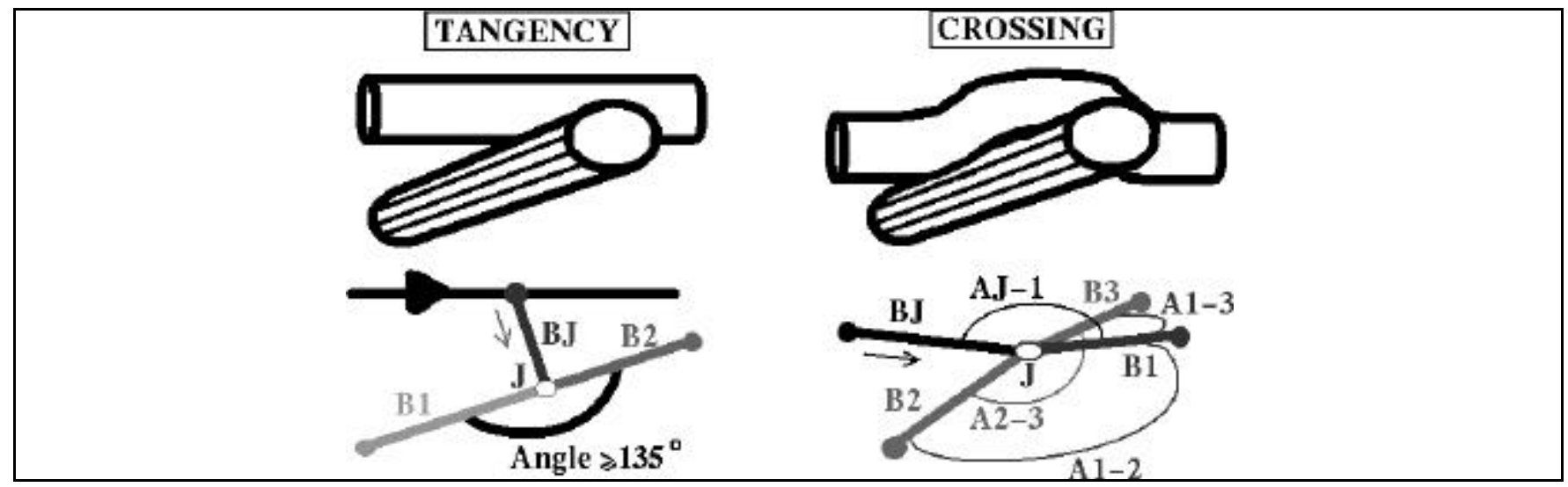

Figure 11.2 type of mistake connection between portal and hepatic veins removed using the angle value between branches.

Finally, this geometrical and topological analyze allows for removing nearly all mistake cases (Fig. 12). 


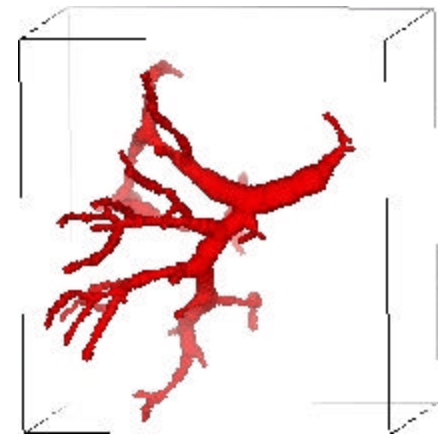

$a$

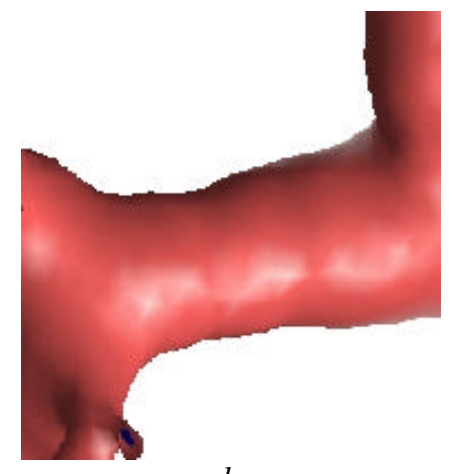

b

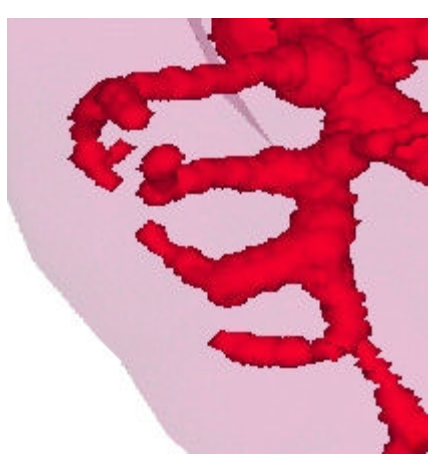

$c$

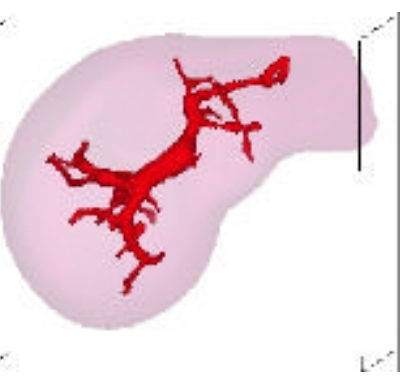

$d$

Figure 12. The four mistake classes correction:

(a) disconnection between portal vein and hepatic vein, (b) disconnection between portal vein and hepatic artery, (c) disconnection of loops in the portal vascular network, (d) removing of false positives.

\section{AUTOMATIC PORTAL VEIN LABELING AND ANATOMICAL SEGMENTATION}

In practice, the current procedure for radiological delineation of anatomical segments is based on the concept of three vertical planes that divide the liver into four segments, and of a transverse scissura that further subdivides the segments into two subsegments each ${ }^{2}$. The three planes are defined from landmarks based on supra-hepatic veins, and the transverse scissura is defined from landmarks based on the portal vein. But, as Fasel et al. ${ }^{2}$ showed, this delineation creates too many errors and must be revised. Moreover, their results show that only procedures that account for the entire portal venous distribution pattern will result in correct depiction of the anatomic reality.

From this conclusion, we defined an anatomical segmentation as the influence area of a set of portal vein branches. According to this definition, the anatomical segmentation becomes a labeling program that consists of merging portal branches in two, five or height sets, with respect to hemiliver, Goldsmith and Woodburne's or Couinaud's segmentation (Fig. 13). Selle et al. ${ }^{15}$ already propose this kind of definition, but their merging method consists in considering the eight major sub-tree into the portal networks. Thus, their system will not be able to correctly label a patient's portal vein after a segmentectomy, or a patient's portal vein with some topological exception as defined by Couinaud ${ }^{4}$.

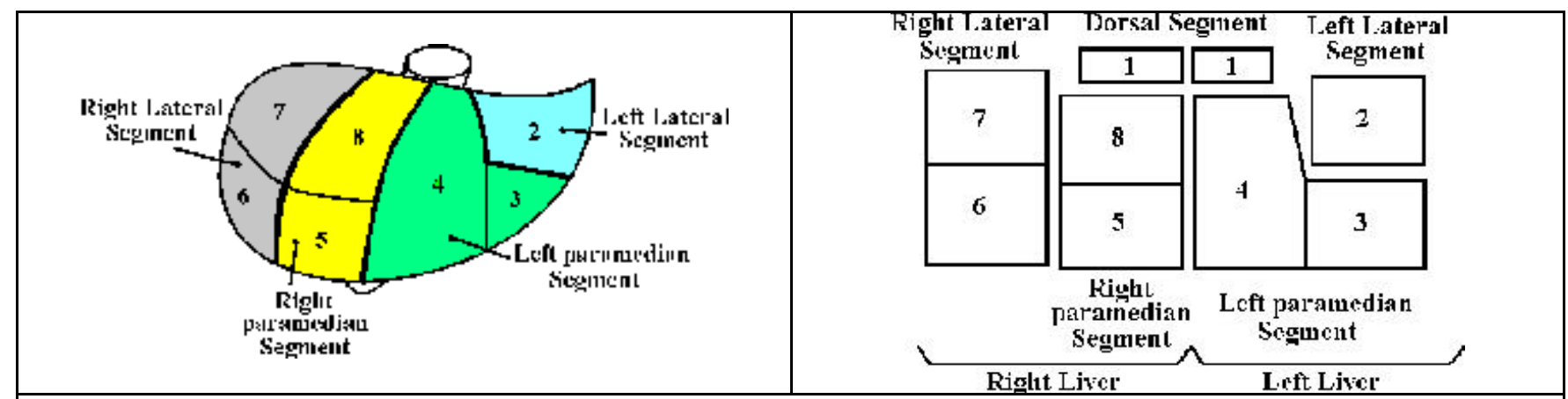

Figure 13. The three anatomical segmentations with respect to hemiliver (right and left liver), Goldsmith \& Woodburne (lateral, paramedian and dorsal right or left segments) and Couinaud (numbers).

We chose to define a new merging system that uses anatomical knowledge translated into topological, geometrical and morphological constraints. This system firstly separates the liver into two hemilivers, secondly separates each hemiliver in three segments (paramedian, lateral and dorsal), and at last separates several segments into subsegment according to the Couinaud's definition. Each of these labelings is performed respectively with the same procedure. Firstly, we compute the influence area in the liver of all branches. We then obtain one volume of hepatic tissue per branch that corresponds to the more precise anatomical sub-segmentation. But, this segmentation is too precise for surgeons, and does not correspond to their usual anatomical segmentation. We then merge these areas by giving the same label to branches having the same origin in the portal tree if the resulting volume of the merging areas verifies some 
constraints translated from definition of anatomical segmentation. These constraints reduce the number of subsegments without merging two anatomical segments with respect to the usual definitions. In order to give to each subsegment the same label as that of the usual definitions, we register an initial segmented model onto the patient's liver using the Montagnat and Delingette's method ${ }^{5}$. We thus obtain a totally automatic labeling and anatomical segmentation of the patient's liver with respect to the three most commonly used anatomical definitions.

\section{RESULTS}

In order to have a quantitative and objective estimation of the quality of our method, we have performed a validation by comparison of our result with the manual delineation of a radiologist. Firstly, where a manual delineation requires more than 11 hours to delineate portal vein and lesions, our method takes only 15 minutes. Comparison of 5 patients shows that our method provides a precision of 2 mm for liver delineation and of less than 1-mm for other anatomical and pathological structures. The use of a deformable liver model obtains good delineation of livers containing large sub capsular tumors that cannot be delineated with current methods. But in five cases among the $35 \mathrm{CT}$-scans, corresponding to patients with atypical liver shape or after segmentectomy, the method was not able to provide a good automatic result, due to liver contours being too far from the initial model. An interactive modification of the shape is then necessary which may then obtain acceptable results, but the time of computation then necessarily increased.

Our automatic lesion segmentation has revealed all hypo-dense lesions over 3-mm of thickness in all of the CT scans evaluated (as compared to the 5-mm usually required by the radiologist). Last, our results show that the automatic portal vein labeling provides exactly the same result as a manual one, including the case of a patient after a segmentectomy.

From these first results, we have then verified on 6 different patients undergoing surgery that reconstruction results of our method before the surgery could precisely guide and improve the surgical procedure. Indeed, in one of the 6 cases, a small lesion of 5.2- $\mathrm{mm}$ of thickness, detected and delineated by our method but missed by the radiologist, totally modified the initial planning (Fig. 14). In two other cases, our anatomical segmentation has accurately localized the patient's tumor to a more precise anatomical segment than the initial preoperative standard landmark-based anatomical segmentation (Fig. 15). This also resulted in a modification of the surgical planning validated intra-operatively and postoperatively through an anapath control. In all cases, clinical validations during surgery have shown that results obtained by our automatic 3D segmentation were correct and add useful information that decreases the time for intra-operative localization of anatomical and pathological structures.

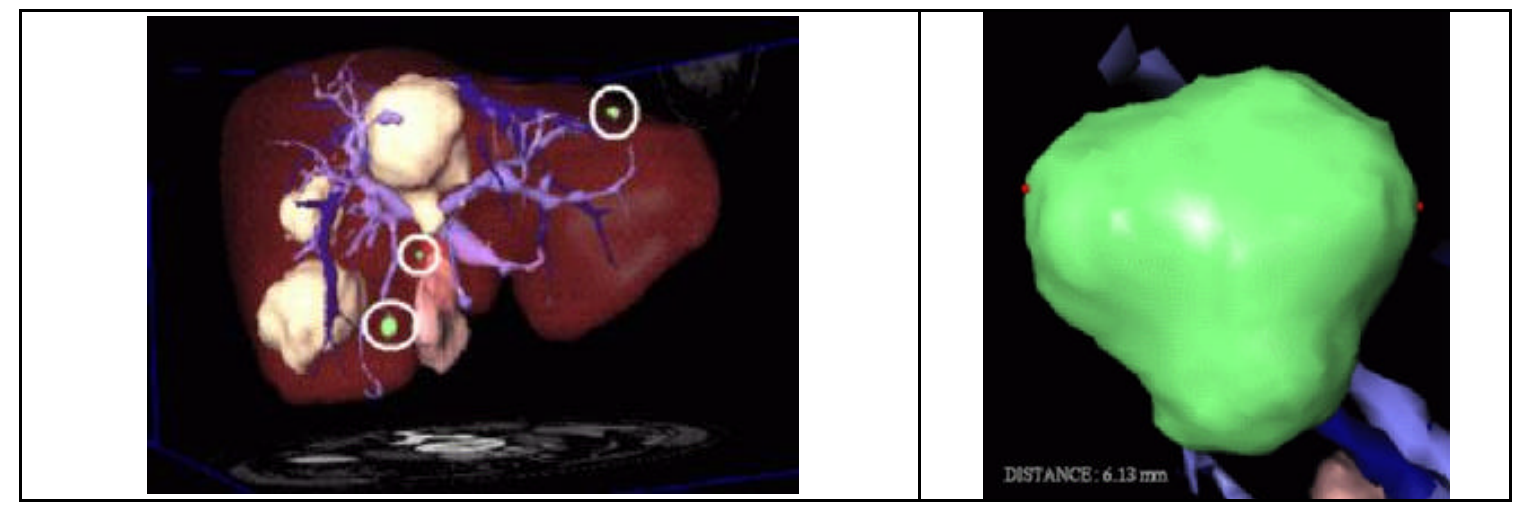

Figure 14. Automatic delineation of tumors shows three small tumors not detected by the radiologist (the encircle ones). Right image shows a zoom onto the left 6-mm thickness tumor. 


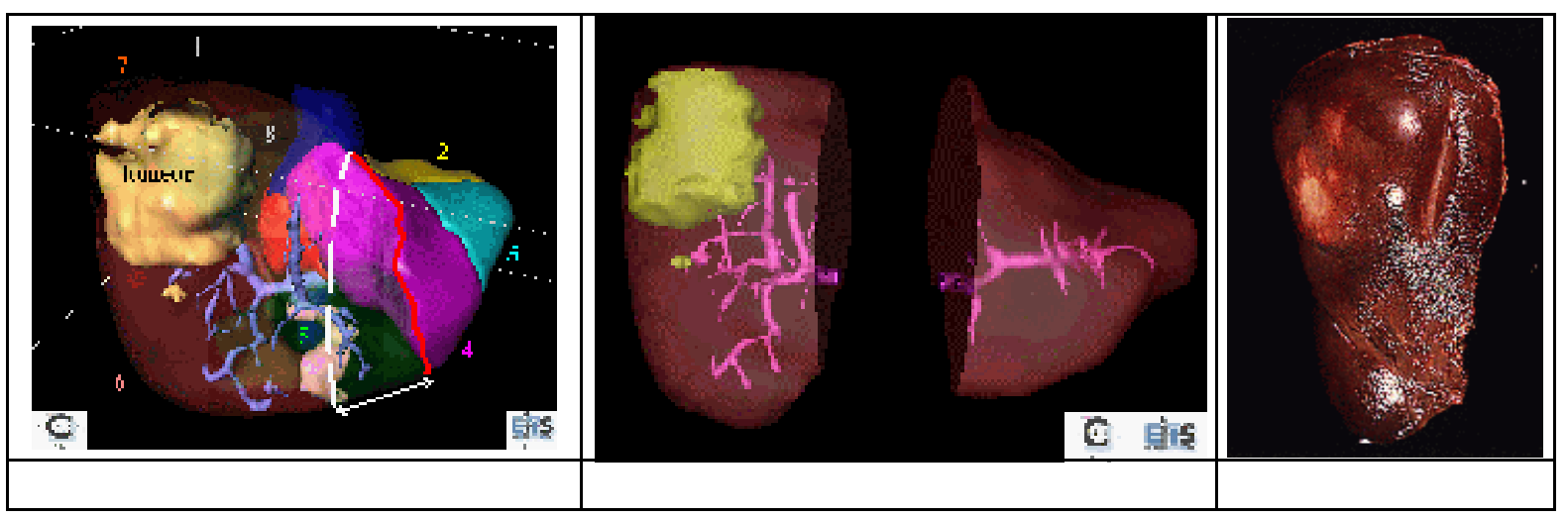

Figure 15. Automatic delineation of a tumor and the anatomical segments: The result shows that the segment 8 contains a part of the tumor which was initially not visible from the CT-scan but verified after surgery.

\section{CONCLUSION}

The originality of this work lies in the full automation of the methods due to original translation of anatomical knowledge into topological and geometrical constraints. The use of deformable models allows thus automatic delineation of livers with large sub-capsular tumors that classical methods do not delineate, but requires interactive modifications for atypical liver shapes (about $15 \%$ of cases). In all other cases, the method offer a fully automatic 3D reconstruction tool for liver surgery, providing not only anatomical and pathological structures visible in the CT scan, but also invisible functional information. It is thus the first complete tool segmenting automatically and simultaneously skin, bones, lungs, kidneys, liver and its tumors, vessels and anatomical segments.

As the first clinical validation seems to show, these original tools could provide real assistance in hepatic surgical planning. Indeed, these techniques detects tumors from only $3 \mathrm{~mm}$ of thickness $(7 \mathrm{~mm}$ less than a classical radiological analysis). It also aids in the intra-operative localization of structures (tumors, anatomical segments).

We need now to perform a larger medical validation in order to confirm these encouraging results and to improve the liver segmentation in order to have an automatic system working with atypical liver shapes. The next step of our work will be then to per-operatively superimpose these $3 \mathrm{D}$ information onto the real patient, providing thus an augmented reality system for liver surgery.

\section{REFERENCES}

1. Fasel J, Gailloud P, Terrier F, Mentha G, Sprumont P (1996) Segmental anatomy of the liver: a review and proposal for an international working nomenclature. European Radiology 6(6): 834-837.

2. Fasel J, Selle D, Evertsz C, Terrier F, Peitgen H, Gailloud P (1998) Segmental Anatomy of the Liver : poor correlation with CT. Radiology 3(206): 151-156.

3. Goldsmith N, Woodburne R (1957) The surgical anatomy pertaining to liver resections. Surgical gynecol Obstet 105: 310-318.

4. Couinaud C (1957), Le foie : études anatomiques et chirurgicales. Masson Edition, France.

5. Montagnat J, Delingette H (1996) Volumetric Medical Images Segmentation using Shape Constrained Deformable Models. CVRMed-MRCAS, Springer Verlag Publisher LNCS 1205, pp 13-22.

6. Gao L, Heath DG, Kuszyk BS, Fishman EK (1996) Automatic Liver Segmentation Techniques for Three-Dimensional Visualization of CT Data. Radiology 2(201): 359-364.

7. Chou JS, Chen SY, Sudakoff GS, Hoffmann KR, Chen CT, Dachman AH (1995) Image fusion for visualization of hepatic vasculature and tumors. Medical Imaging 1995: Image Processing, SPIE Proceedings 2434, pp. 157-163.

8. Bae KT, Giger ML, Chen CT, Kahn CE (1993) Automatic segmentation of liver structure in CT images. Medical Physics 1(20): 71-78. 
9. Matsushita S, Oyamada H, Kusakabe M, Suzuki N (1993) Attempt to extract 3-D image of liver automatically out of Abdominal MRI. Medical Imaging 1993: Image Processing, SPIE Proceedings 1898, pp 803-808.

10. Inaoka N, Suzuki H, Fukuda M (1992) Hepatic Blood Vessels Recognition using Anatomical Knowledge. Medical Imaging 1992: Image Processing, SPIE Proceedings 1652, pp 509-513.

11. Masutani Y, Yamauchi Y, Suzuki M, Ohta Y, Dohi T, Tsuzuki M, Hashimoto D (1995) Development of interactive vessel modelling system for hepatic vasculature from MR images. Medical and Biomedical Engineering and Computing 1(33): 97-101.

12. Zahlten C, Jürgens H, Evertsz CJG, Leppek R, Peitgen HO, Klose KJ (1995) Portal Vein Reconstruction Based on Topology. European Journal of Radiology 2(19): 96-100.

13. Zahlten C, Jürgens H, Peitgen HO (1995) Reconstruction of branching blood vessels from CT-data. Workshop of Visualization in Scientific Computing, Springer Verlag Publisher Eurographics 1995, pp 41-52.

14. Selle D, Schindewolf T, Evertsz CJG, Peitgen HO (1998) Quantitative analysis of CT liver images. International workshop on Computer Aided Diagnosis in Medical Imaging, ICS 1182, Chicago.

15. Bellon E, Feron M, Maes F, Van Hoe L, Delaere D, Haven F, Sunaert S, Baert AL, Marchal G, Suetens P (1997) Evaluation of manual vs semi-automated delineation of liver lesions on CT images. European Radiology 3(7): 432-438.

16. Kovalev VA (1995) Rule-Based Method for tumor Recognition in Liver Ultrasonic Images. Image Analysis and Processing, Springer Verlag Publisher LNCS 974, pp 217-222.

17. Brown LG (1994) A Survey of Image Registration Techniques. ACM Computing Surveys 4(24): 325376.

18. McInerney T, Terzopoulos D (1993) A Finite Element Model for 3D Shape Reconstruction and Nonrigid Motion Tracking. International Conference on Computer Vision, Berlin, IEEE Computer Society Press, pp. 518-523.

19. Kass M, Witkin A, Terzopoulos D (1987) Snakes: Active Shape Models. International Journal of Computer Vision 4(1): 321-331.

20. Krissian K, Malandain G, Ayache N (1996) Directional Anisotropic Diffusion Applied to Segmentation of Vessels in 3D Images. INRIA France, RR-3064.

21. Press WH, Flannery BP, Teukolsky SA, Vetterling WT (1998) Numerical Recipes in C. Cambridge University Press.

22. Bammer R, Stollberger R, Pedevilla M, Ropele S, Ebner F, Wach P (1997) Automated Tissue Classification of Extremities Using Knowledge-Based Segmentation of MR Images. Computer Assisted Radiology and Surgery, Elsevier Science B.V. Publisher, pp 252-258.

23. Atkins MS, and Mackiewich BT (1996) Automatic Segmentation of the Brain in MRI. Visualization in Biomedical Computing 1996, Springer Verlag Publisher LNCS 1131, pp 241-246.

24. Goshtasby A, O'Neill WD (1994) Curve Fitting by a Sum of Gaussians. CVGIP: Graphical Models and Image Processing 4(56): 281-288.

25. Brummer ME, Mersereau RM, Eisner RL, Lewine RJ (1993) Automatic Detection of Brain Contours in MRI Data Sets. IEEE Trans. on Medical Imaging 2(12): 153-166.

26. Bellocq JP, Marcellin L, Chenard-Neu MP (1992) Anatomie pathologique des métastases hépatiques des adénocarcinomes colorectaux. Traitement des métastases hépatiques des cancers colorectaux, Springer-Verlag Publisher, pp 11-25.

27. Bertrand G, Malandain G (1994) A new characterization of three-dimensional simple points. Pattern Recognition Letters 2(15): 169-175.

28. Malandain G, Bertrand G, Ayache N (1993) Topological Segmentation of Discrete Surfaces. International Journal of Computer Vision 2(10): 183-197. 